\title{
Comunicação
}

[Communication]

\section{Eritrograma e variantes de hemoglobina em caprinos da raça Canindé}

\author{
[Erythrogram and hemoglobin types in Canindé goats] \\ D.O. Luz $^{1}$, R.M. Lacerda ${ }^{1}$, R.A. Barreto Júnior ${ }^{2}$, B. Soto-Blanco ${ }^{2^{*}}$ \\ ${ }^{1}$ Aluno de graduação - DCAn/UFERSA - Mossoró, RN \\ ${ }^{2}$ Departamento de Ciências Animais - UFERSA \\ 59625-900 - Mossoró, RN
}

O estudo do polimorfismo da hemoglobina possui significância para a determinação de variantes presentes na população avaliada, bem como para o diagnóstico de variantes patológicas (Alves et al., 2003). Em animais, o tipo de hemoglobina pode apresentar relação com a resistência a helmintos (Buvanendran et al., 1981), com a intensidade da resposta imune (Cuperlovic et al., 1978) e com parâmetros produtivos, como eficiência reprodutiva e produção de lã e leite (Dally et al., 1980). Além disso, a determinação das variantes de hemoglobina pode ser um dos parâmetros genéticos empregados em estudos de identificação de indivíduos e de parentesco (Lacerda e Soto-Blanco, 2006).

São escassos os trabalhos realizados com o objetivo de avaliar a variabilidade da hemoglobina em caprinos (Huisman et al., 1969; Johnson et al., 2002), sendo mais marcante em raças nativas do Brasil. Isto é bastante relevante, pois a criação de raças nativas de caprinos vem adquirindo importância econômica e social na região Nordeste do Brasil, pela sua característica de apresentar rusticidade e se adaptar muito bem ao clima da região. Uma das raças nativas é a Canindé, originada de caprinos trazidos pelos colonizadores portugueses na época da colonização. São animais de grande rusticidade, alta prolificidade e com características fenotípicas definidas. Estão sendo utilizados em cruzamentos com reprodutores de raças exóticas, com características fenotípicas semelhantes, visando à obtenção de cabras de grande produção de leite, mas com melhores condições de adaptação ao meio (Medeiros et al., 1994).

O presente trabalho teve por objetivo determinar o eritrograma e as variantes de hemoglobina em caprinos da raça Canindé.

Foram utilizadas 20 amostras de sangue de caprinos da raça Canindé considerados clinicamente sadios, fêmeas, com aproximadamente dois anos de idade, procedentes do município de Angicos, RN. As amostras de sangue foram colhidas por meio de punção da veia jugular em tubos de vidro contendo EDTA. As amostras foram levadas imediatamente após a coleta ao laboratório, onde foram realizados os seguintes exames: hematócrito, contagem do número de hemácias, dosagem da hemoglobina e separação de hemoglobina por eletroforese, e realizados os cálculos dos índices hematimétricos absolutos volume corpuscular médio (VCM), hemoglobina corpuscular média (HCM) e concentração de hemoglobina corpuscular média (CHCM). A separação eletroforética das hemoglobinas foi realizada com o auxílio do Sistema para Eletroforese Celm SE-250, utilizando o kit CelmGel Hemoglobina Alcalina.

Os resultados são apresentados na forma de média e seus respectivos desvios-padrão. As médias dos valores obtidos nos animais, agrupados segundo o tipo de hemoglobina, foram comparadas utilizando a análise de variância de uma via, seguida pelo teste de comparações

Recebido em 12 de janeiro de 2009

Aceito em 28 de dezembro de 2009

* Autor para correspondência (corresponding author)

E-mail: soto-blanco@ufersa.edu.br 
múltiplas pareadas de Dunn. O teste de correlação de Pearson foi utilizado para avaliação de correlação entre os diferentes parâmetros do eritrograma. O nível de significância estatística foi estabelecido como $\mathrm{P}<0,05$, e a análise estatística foi realizada com o auxílio do programa software Sigma Stat versão 1.0 .
Os valores do eritrograma dos caprinos são apresentados na Tab. 1 e são semelhantes aos apresentados na literatura (Viana et al., 2003; Mattos et al., 2005). Assim, não é necessário estabelecer padrões específicos para eritrograma da raça Canindé, uma vez que os adotados para caprinos em geral são adequados.

Tabela 1. Eritrograma (média \pm desvio-padrão de caprinos da raça Canindé

\begin{tabular}{lcc}
\multicolumn{1}{c}{ Parâmetro } & Média \pm desvio-padrão & Valores de referência $^{1}$ \\
\hline Eritrócitos $\left(\times 10^{6} / \mu \mathrm{L}\right)$ & $15,3 \pm 4,54$ & $8,0-18,0$ \\
Hemoglobina $(\mathrm{g} / \mathrm{dl})$ & $10,1 \pm 1,05$ & $8,0-12,0$ \\
Hematócrito $(\%)$ & $34,6 \pm 4,62$ & $22-38$ \\
VCM (fL) & $24,0 \pm 5,83$ & $15-25$ \\
HCM $(\mathrm{pg})$ & $7,0 \pm 1,71$ & $5,2-8,0$ \\
CHCM $(\%)$ & $29,3 \pm 2,44$ & $30-36$ \\
\hline
\end{tabular}

${ }^{1}$ Kramer (2006)

VCM: volume corpuscular médio; HCM: hemoglobina corpuscular média; CHCM: concentração de hemoglobina corpuscular média.

A eletroforese das hemoglobinas revelou uma banda rápida caracterizada como hemoglobina $\mathrm{A}$ (HbAA). Em caprinos, já foram descritas as variantes homozigotas $\mathrm{HbAA}$ e $\mathrm{HbBB}$ e a heterozigota $\mathrm{HbAB}$ (Huisman et al., 1969; Johnson et al., 2002), além de outra variante denominada HbD (Huisman et al., 1969). Diversas raças caprinas apresentam apenas a variante $\mathrm{HbAA}$, enquanto outras a $\mathrm{HbAA}$ e a HbAB (Johnson et al., 2002). A variabilidade das hemoglobinas foi mais estudada em ovinos do que em caprinos. Alguns estudos realizados em ovinos encontraram as variantes $\mathrm{HbAA}, \mathrm{HbBB}$ e HbAB (Sotomaior e Thomaz-Soccol, 1998; Lacerda e Soto-Blanco, 2006). Em ovinos das raças iranianas Baloochi e Kordi, foram encontradas apenas $\mathrm{HbAA}$ e $\mathrm{HbAB}$ e não a HbBB (Mohri et al., 2005). Ainda, há raças de ovinos que apresentam $\mathrm{HbBB}$ e $\mathrm{HbAB}$, mas não HbAA (Mostaghni, 1978). Essas diferenças nas frequências das variantes de hemoglobina estão claramente associadas às diferenças raciais. Além disso, as variantes da hemoglobina podem ser ferramentas importantes para a determinação de variabilidade genética e de parentesco (Lacerda e Soto-Blanco, 2006). Nesse sentido, a ocorrência de apenas uma variante de hemoglobina nos caprinos avaliados indica a ocorrência de baixa variabilidade genética no rebanho.

A baixa variabilidade genética encontrada neste estudo, se for comprovada em outros estudos com maior número de animais, poderá orientar futuros programas direcionados ao cruzamento com outras raças.

Palavras-chave: caprino, raça Canindé, hemoglobina, eritrograma

\begin{abstract}
The erythrogram and hemoglobin types in Canindé goats were determined. Twenty blood samples were collected from the animals and used for determination of packed cell volume (PCV), erythrocytes counts, hemoglobin concentration, calculation of mean corpuscular volume (MCV) and mean corpuscular hemoglobin concentration (MCHC), and electrophoretic separation of hemoglobin types. The values for erythrogram were: PCV 34.6 $\pm 4.62 \%$, hemoglobin $10.1 \pm 1.05 \mathrm{mg} / \mathrm{dl}$, erythrocytes $15.3 \pm 4.54 \times 10^{6} / \mathrm{mm}^{3}$, $M C V 24.0 \pm 5.83 \mathrm{fl}$, and MCHC 29.3 $2.44 \mathrm{~g} / \mathrm{dl}$. Electrophoretic separation of hemoglobin types revealed only one type characterized as hemoglobin A (HbAA).
\end{abstract}

Keywords: goat, Canindé breed, hemoglobin, erythrogram 


\section{REFERÊNCIAS BIBLIOGRÁFICAS}

ALVES, R.T.; MATTOS, L.C.; FERRARI, F. et al. Avaliação do polimorfismo de grupos sanguíneos e fenótipo de hemoglobinas em um grupo de universitários de São José do Rio Preto, SP. Rev. Bras. Hematol. Hemoter., v.25, p.65-71, 2003.

BUVANENDRAN, V.; SOORIYAMOORTHY, T.; OGUNSUSI, R.A. et al. Haemoglobin polymorphism and resistance to helminths in red Sokoto goats. Trop. Anim. Health Prod., v.13, p.217-221, 1981.

CUPERLOVIC, K.; ALTAIF, K.I.; DARGIE, J.D. Genetic resistance to helminths: a possible relationship between haemoglobin type and the immune response of sheep to non-parasitic antigens. Res. Vet. Sci., v.25, p.125-126, 1978.

DALLY, M.R.; HOHENBOKEN, W.; THOMAS, D.L. et al. Relationships between hemoglobin type and reproduction, lamb, wool and milk production and health-related traits in crossbred ewes. J. Anim. Sci., v.50, p.418-427, 1980.

HUISMAN, T.H.J.; LEWIS, J.P.; BLUNT, H.M. et al. Hemoglobin $\mathrm{C}$ in newborn sheep and goats: a possible explanation for its function and biosynthesis. Pediatr Res., v.3, p.189-198, 1969.

JOHNSON, E.H.; NAM, D.; AL-BUSAIDY, R. Observations on haemoglobin types in three breeds of omani goats. Vet. Res. Commun., v.26, p.353-359, 2002.

KRAMER, J.W. Normal hematology of cattle, sheep, and goats. In: FELDMAN, B.F.; ZINKL, J.G.; JAIN, N.C. (Ed.) Schalm's veterinary hematology, 5.ed. Ames: Blackwell, 2006. p.1075-1084.
LACERDA, R.M.; SOTO-BLANCO, B. Determinação das variantes de hemoglobina em ovinos mestiços Santa Inês. Caatinga, v.19, p.345-349, 2006.

MATTOS, M.J.T.; OLIVEIRA, C.M.B.; LUSTOSA, A. et al. Influência do parasitismo por nematódeos sobre o perfil hematológico de caprinos. Arq. Bras. Med. Vet. Zootec., v.57, p.133-135, 2005.

MEDEIROS, L.P.; GIRÃO, R.N.; GIRÃO, E.S. et al. Caprinos: princípios básicos para sua exploração. Teresina: Embrapa-Meio Norte, 1994. 177p.

MOHRI, M.; JANNATABADI, A.A.; ASLANI, M.R. Studies on haemoglobin polymorphism of two breeds of Iranian sheep and its relationship to concentrations of iron, copper, haemoglobin and RBC number. Vet. Res. Commun., v.29, p.305-312, 2005.

MOSTAGHNI, K. Studies on haemoglobin variants and some blood minerals of different breeds of Iranian sheep. J. Vet. Med. A, v.25, p.647-651, 1978.

SOTOMAIOR, C.S.; THOMAZ-SOCCOL, V. Estudo do tipo de hemoglobina como auxiliar na seleção de ovinos resistentes e susceptíveis aos helmintos gastrintestinais. Arch. Vet. Sci., v.3, p.51-55, 1998.

VIANA, R.B.; BIRGEL JUNIOR, E.H.; AYRES, M.C.C. et al. Influência da gestação e do puerpério sobre o eritrograma de caprinos (Capra hircus) da raça Saanen, criados no Estado de São Paulo. Braz. J. Vet. Res. Anim. Sci., v.40, p.178-184, 2003. 